\title{
UMA NOVA FERRAMENTA BASEADA NA TRANSFORMADA WAVELET PARA LOCALIZAÇÃO DIGITAL DE FALTAS
}

\author{
Murilo da Silva* \\ Mário Oleskovicz* \\ murisilvalsel. eesc.usp.br \\ Denis V. Coury* \\ couryesel.eesc.usp.br \\ olesk@sel.eesc.usp.br \\ * Departamento de Engenharia Elétrica, Escola de Engenharia de São Carlos, Universidade de São Paulo - Av. Trabalhador \\ Sancarlense, 400 - 13566-590, São Carlos, São Paulo, Brasil
}

\begin{abstract}
This work presents an application of Wavelet Transform (WT) to analyze the high frequency transients in a transmission system generated by a fault situation, with the purpose of determining its accurate location. The methodology of fault location used is based on the traveling waves theory, where, in function of the propagation time of the signals between fault point and the terminals of the line utilizing WT, the fault location is determined. The fault location problem is implemented using Matlab software. In this implementation, the user can choose the fault location technique to be used: using registered data in one or in both terminals of the line, depending on his necessity and/or availability of the required resources. Independent of the considered technique, the implemented algorithm presents promising results to the fault location on the different transmission systems. It was possible to overcome some of the limitations imposed by the traditional fault locators.
\end{abstract}

KEYWORDS: Fault Location, Transmission Lines, Power System, Traveling Waves, Wavelet Transform.

\section{RESUMO}

Este trabalho apresenta uma aplicação da Transformada Wa-

Artigo submetido em 03/08/2004

1a. Revisão em 09/09/2005;

Aceito sob recomendação do Ed. Assoc. Prof. Glauco Taranto velet (TW) para analisar os transitórios de alta freqüência em um sistema de transmissão gerados por uma situação de falta, tendo como principal objetivo, a determinação do ponto de ocorrência da mesma, o mais precisamente possível. A abordagem baseia-se na teoria de ondas viajantes, onde, em função do tempo de propagação do sinal entre o ponto de falta e os terminais da linha, revelados pela TW, a localização da falta é estimada. Desta tomada de tempo, obtém-se a indicação da localização de uma determinada situação de falta pela implementação prática de um algoritmo computacional, dispondo-se do software MatLab?. Nesta implementação, o usuário pode escolher dentre as técnicas de localização a ser utilizada, de modo a trabalhar com dados registrados em um ou em ambos os terminais da linha, conforme sua necessidade e/ou disponibilidade dos recursos requeridos. Independente da técnica de localização utilizada, o algoritmo desenvolvido apresenta resultados promissores para localização de faltas sobre diferentes sistemas de transmissão, além de superar algumas limitações impostas aos localizadores de faltas tradicionais.

PALAVRAS-CHAVE: Localização de Faltas, Linhas de Transmissão, Sistemas de Potência, Ondas Viajantes, Transformada Wavelet.

\section{INTRODUÇÃO}

A energia elétrica é um dos recursos fundamentais para o desenvolvimento econômico de um país, bem como, para pro- 
mover a satisfação e o bem-estar da sociedade. Dessa forma, os Sistemas Elétricos de Potência (SEP) devem garantir um alto grau de confiabilidade na continuidade do fornecimento de energia elétrica. Contudo, interrupções no fornecimento de energia podem ser provocadas pela ocorrência de diferentes tipos de fenômenos eletromagnéticos que podem afetar distintas classes de consumidores. Situações faltosas podem ocorrer nos diversos componentes de um SEP, dentre os quais podemos destacar as Linhas de Transmissão (LTs) como o elemento mais susceptível, especialmente se considerarmos suas dimensões físicas, complexidade funcional e o ambiente em que se encontram, apresentando uma maior dificuldade para manutenção e monitoramento.

O crescente aumento em complexidade dos SEP devido a demanda de energia e a interligação dos sistemas existentes, tem exigido uma considerável melhora dos equipamentos de controle, proteção, bem como de localização de faltas. Desta melhora nos dispositivos empregados, busca-se possibilitar uma redução do período de interrupção do fornecimento de energia, em caso de falhas, e garantir uma operação confiável e econômica do sistema de potência, seja em condições normais de operação ou em contingência. Como exemplo, o crescimento da competição no mercado de energia nacional, devido ao processo de desestatização pelo qual vem passando o setor elétrico, tem levado as empresas de energia elétrica a investirem em modernos sistemas de gerenciamento e proteção, a fim de assegurar uma fatia significativa do mercado, através da oferta de energia a um menor custo e com alto padrão de qualidade.

Nas últimas duas décadas, utilizar dispositivos baseados em microprocessadores para as soluções de problemas relacionados aos sistemas elétricos de potência, tem sido assunto de grande interesse por parte de engenheiros e pesquisadores. Esse interesse se deve ao rápido avanço da tecnologia, aliado ao desenvolvimento na área de software destinado às aplicações em sistemas elétricos de potência, especialmente quando se faz necessário grande precisão, como é a situação da localização de faltas.

\subsection{Localizadores de Falta para Linhas de Transmissão}

Um localizador digital de faltas, como o próprio nome induz, tem por objetivo determinar, o mais precisamente possível, o ponto de ocorrência de uma falta em uma linha de transmissão, o que é de suma importância para uma operação confiável e econômica do sistema de potência. Além da segurança do sistema, conhecendo-se a localização da falta, um menor tempo é despendido nos serviços de manutenção e reparo, o que permite uma rápida restauração da operação às condições normais desejadas. Sendo assim, além de prover a localização de faltas sustentadas, um localizador pode vir a estimar faltas transitórias, como por exemplo, descargas atmosféricas, podendo alertar para possíveis pontos fracos nos sistemas de transmissão, possibilitando que medidas preventivas sejam executadas para evitar futuros problemas de maior grandeza.

Como já comentado, o desenvolvimento de novas técnicas de localização digital de faltas para linhas de transmissão, utilizando dispositivos baseados em microprocessadores, tem sido um assunto de grande interesse para pesquisadores e engenheiros da área de potência nos últimos anos. Várias abordagens foram desenvolvidas e diferentes princípios já foram aplicados ao problema da localização de faltas em linhas de transmissão. Basicamente, os métodos de localização de faltas podem ser classificados em duas categorias: (a) nos métodos baseados em componentes de freqüência fundamental e (b) nos métodos baseados em componentes de alta freqüência gerados por uma falta.

Os métodos que se baseiam em componentes fundamentais, geralmente determinam a localização da falta caracterizando a impedância da linha entre o relé e o ponto de falta. Dependendo da caracteristica peculiar a cada uma das variações destes métodos, estes podem ser mais ou menos afetados por diversos fatores, tais como: resistência de falta, contribuição de corrente do terminal remoto, carregamento da linha, parâmetros da fonte, etc (Coury e Gautier, 2000) e (Aggarwal et al., 1993).

A segunda abordagem, referenciado como métodos das ondas viajantes, baseia-se na determinação do intervalo de tempo de viagem, da onda de tensão ou corrente, do ponto da falta ao terminal da linha onde está instalado o localizador e na velocidade de propagação desta onda na linha, para estimar a distância da falta. Este método supera as dificuldades encontradas pela aplicação do método baseado em componentes de freqüência fundamental conforme apresentado por Bo e colegas (Bo et al., 2000). A grande limitação relacionada com o método baseado em ondas viajantes, reside na necessidade de se trabalhar com altas taxas de amostragem. Contudo, visto o enorme desenvolvimento dos conversores analógicos/digitais (A/D) de alta velocidade e da tecnologia dos transdutores de corrente e tensão capazes de registrar os transitórios oriundos de uma falta com altas taxas de amostragem, a sua aplicação tem-se tornado viável. Como exemplos de aplicações decorrentes deste avanço, citamos Bo et al. (1997), Magnago e Abur (1998), Gale et al. (2001), Zhang et al. (2003) e Silva et al (2004).

Recentemente, há propostas do emprego de ferramentas inteligentes, como por exemplo, Redes Neurais Artificiais (RNAs), ao problema de localização de faltas como em Purushothama et al, 2001. Neste trabalho em específico, abordou-se o problema da localização de faltas através da 
implementação prática de um algoritmo computacional baseado na teoria de ondas viajantes e no uso da Transformada Wavelet (TW). A TW é utilizada devido a sua capacidade de detectar com precisão, tanto no domínio do tempo, como da freqüência, a presença de distúrbios presentes em um dado sinal, característica essa que a torna muito adequada para ser aplicada ao problema delineado.

A metodologia apresentada neste trabalho é baseada na teoria das ondas viajantes para detecção do intervalo de tempo de viagem da onda do ponto de falta ao terminal ou terminais da linha em questão, a qual será apontado pelo emprego da TW, para estimação da distância da falta. Como mencionado, o algoritmo de localização foi desenvolvido de maneira a possibilitar a localização da falta utilizando dados registrados em um ou em ambos os terminais da linha. Isso vem a possibilitar uma maior flexibilidade ao usuário, podendo o mesmo escolher a técnica de localização a ser utilizada, de acordo com suas necessidades e os recursos disponíveis no sistema.

Na estrutura do presente trabalho encontram-se, nesta ordem, apontamentos sobre a teoria de ondas viajantes e da Transforma Wavelet, o método de localização desenvolvido, testes e resultados obtidos, finalizando com algumas conclusões gerais.

\section{CONCEITOS BÁSICOS}

Esta seção tem como objetivo principal a apresentação de forma simples e objetiva da teoria básica sobre ondas viajantes e da transformada wavelet.

\subsection{A Teoria de Ondas Viajantes}

Quando falamos em ondas viajantes, estamos nos referindo as formas de ondas de propagação da energia sobre um sistema, em nosso caso, da propagação da energia elétrica sobre uma linha de transmissão. Se houver uma variação qualquer de corrente ou de tensão em um terminal de uma dada linha de transmissão, o outro terminal só irá sentir tal variação, quando a onda referente ao sinal elétrico percorrer todo o comprimento da mesma. Portanto, o terminal remoto da linha de transmissão, não pode influenciar nas decisões sobre o sistema, até que a onda tenha se deslocado da fonte do terminal local ao terminal remoto, onde, através da interação deste com a linha de transmissão, será produzida uma resposta (forma de onda) que retornará para a fonte local. Desta maneira, os sinais elétricos tendem a se propagar para frente e para trás, como ondas viajantes, normalmente dissipando energia devido as perdas na linha (Greenwood, 1971).

Do exposto acima, temos que qualquer distúrbio em uma linha de transmissão (tal como os provocados por descargas atmosféricas, curtos-circuitos, ou uma alteração da condição de regime permanente) dá origem a ondas viajantes, as quais representam uma composição de frequência, que pode variar de poucos kilohertz a diversos megaherts Estas por sua vez, deslocam-se no sentido das extremidades da linha de acordo com a velocidade de propagação característica da mesma. Quando estas ondas viajantes encontram certas descontinuidades físicas do sistema, estas descontinuidades provocam a sua reflexão ou refração. Este sucessivo processo continua até que as ondas sejam atenuadas e ou extintas devido às perdas na linha, alcançando o regime permanente do distúrbio.

Quando uma onda viajante atinge uma descontinuidade, ou seja, um ponto de transição no qual há uma súbita mudança nos parâmetros do circuito, tais como num terminal aberto, uma junção com uma outra linha, um enrolamento de uma máquina ou transformador, etc, uma parte da onda é refletida para trás, e outra parte, transmitida para a sessão seguinte do circuito. A onda que chega na descontinuidade é chamada de onda incidente e as duas ondas oriundas da descontinuidade são chamadas de ondas refletidas e refratadas, respectivamente. Tais ondas formadas no ponto de transição seguem as leis de Kirchhoff. Elas satisfazem a equações diferenciais das linhas de transmissão, e obedecem os princípios de conservação de energia (Bewley, 1933).

$\mathrm{O}$ algoritmo desenvolvido neste trabalho baseia-se no princípio das ondas viajantes comentado. Pela implementação desenvolvida, determinando-se o intervalo de tempo de viagem da onda do ponto de falta ao terminal ou terminais da linha, considerando a velocidade de propagação da mesma, estima-se então o ponto exato de ocorrência da situação faltosa.

\subsection{A Tranformada Wavelet}

A análise de dados de acordo com escalas variáveis no domínio do tempo e da frequiência é a idéia básica da utilização da teoria Wavelet. O termo "Wavelet" foi originariamente introduzido por J. Morlet, tendo a base matemática de suas idéias formalizada pelo físico teórico Alex Grossmann (Daubechies, 1996).

A Transformada Wavelet (TW) é uma ferramenta matemática para análise de sinais similar à Transformada de Fourier (TF). A TW decompõe o sinal em diferentes escalas, com diferentes níveis de resolução, a partir de uma única função. Diferentemente da TF que fornece uma representação global do sinal, a TW apresenta representações locais no domínio do tempo e da frequiência de um dado sinal (Santoso et al., 1996).

A Transformada Wavelet Contínua (TWC) de dada função $f$ 


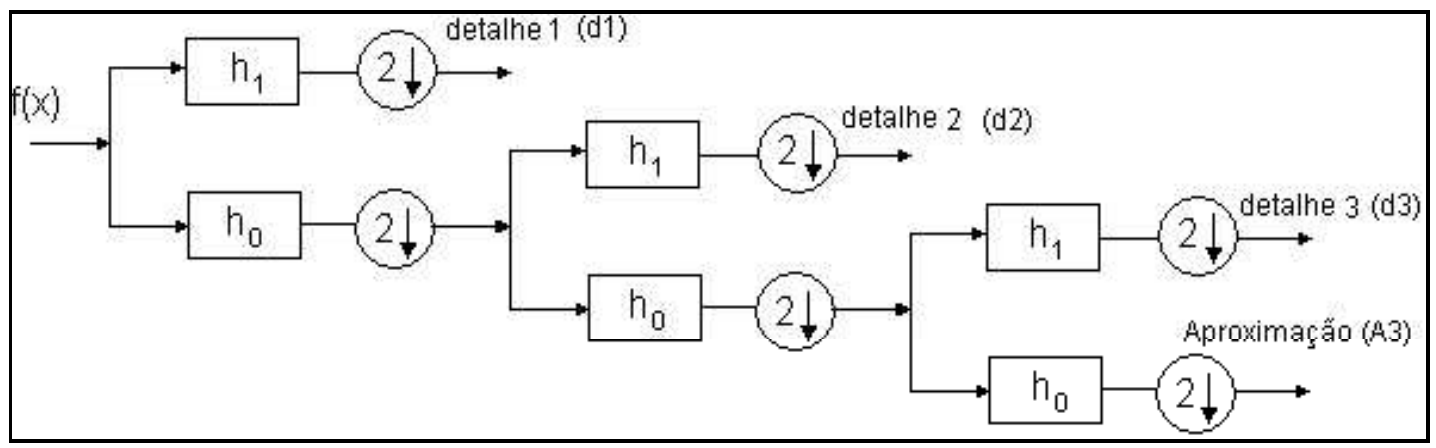

Figura 1: Processo de decomposição de um sinal em AMR

é dada pela equação:

$$
(T W C \cdot)(a, b)=\int f(t) \overline{\psi_{a, b}(t)} d t
$$

Nesta equação, os parâmetros $a$ e $b$ variam continuamente em $R$, sendo as funções $\Psi$ a,b são denominadas Wavelets e definidas de acordo com a Eq. (2):

$$
\psi_{a, b}(t)=\frac{1}{\sqrt{a}} \psi\left(\frac{t-b}{a}\right)
$$

Analisando a Eq. (1) pode-se perceber que a Transformada Wavelet depende de dois parâmetros, $a$ e $b$, que correspondem, respectivamente, às informações da escala, ou dilatação, e translação, ou tempo. Tal equação decompõe $f$ em uma superposição de Wavelets $\Psi \mathrm{a}, \mathrm{b}$, sendo os coeficientes nesta superposição obtidos pela transformada Wavelet de $f$.

A análise por Wavelet transpõe as limitações dos métodos de Fourier pelo emprego de funções de análise (wavelets) que são locais, no tempo e na frequiência. A transformada wavelet é muito bem aceita para uma ampla faixa de sinais que não são periódicos e que podem conter ambos os componentes senoidais e de impulso, como é típico nos transitórios de sistemas de potência. Em particular, a habilidade da Wavelet em se concentrar em pequenos intervalos de tempo, para componentes de alta freqüência, e em longos intervalos de tempo, para componentes de baixa frequiência, melhora a análise de sinais caracterizados por impulsos e oscilações localizadas, particularmente na presença da componente fundamental e dos componentes harmônicos de baixa ordem. Para esta análise em específico, a TW pode apresentar uma janela que automaticamente se ajusta para proporcionar a resolução desejada. A TW pode ser encontrada em duas formas: (i) a Transformada Wavelet Continua (TWC) e (ii) a Transformada Wavelet Discreta (TWD) (Hwan e Aggarwal (2000)).

Uma das formas para implementação da TWD consiste no sucessivo agrupamento de pares de filtros passa baixa e passa alta em cada estágio de escala da TW. Isto pode ser visto como sucessivas aproximações da mesma função, onde cada aproximação ressalta importantes informações relativas a uma dada escala (faixa de frequiência). Este processo de filtragem é melhor representado pela técnica da Análise Multiresolução (AMR). Tal processo baseia-se na filtragem de um sinal a ser analisado através de bancos de filtros passa alta e passa baixa, fornecendo versões do sinal original relativas aos sinais de aproximação e detalhe. A aproximação compreende as baixas frequiências do sinal original, enquanto o detalhe engloba o conteúdo de alta frequiência do sinal original. Dessa forma, o sinal original é dividido em diferentes escalas de resolução, particularmente em diferentes faixas de freqüências, como no caso da análise de Fourier.

Em suma, a idéia básica do processo de AMR é dividir o espectro de um dado sinal em sub-bandas de freqüência e então tratar individualmente cada uma das sub-bandas, considerando o propósito desejado, uma vez que cada nível de decomposição conterá importantes informações em frequiência e no tempo, relativas ao sinal original.

A Figura 1 ilustra um processo de análise multiresolução em três níveis de resolução. Os detalhes e aproximações do sinal original $f(x)$ são obtidos por meio de bancos de filtros, os quais são formados por filtros passa-baixa $\left(\mathrm{h}_{0}\right)$ e passa-alta $\left(\mathrm{h}_{1}\right)$ conforme já comentado. Um filtro passa-baixa remove os componentes de altas freqüências, enquanto o filtro passa alta separa o conteúdo de alta freqüência no sinal sendo analisado.

Dentre as principais características da TW pode-se destacar a sua habilidade em analizar o sinal no domínio do tempo e freqüência simultaneamente. Outra habilidade da TW é de focar pequenos invervalos de tempo em altas frequencias e longo intervalos de tempo para baixa frequencias, o que possibilita uma melhor análise do sinal. A TW também possuim um janelamento que se adapta automaticamente permitindo uma melhor resolução. Estas características permitem que a TW seja utilizada em um algoritmo de localização de fal- 


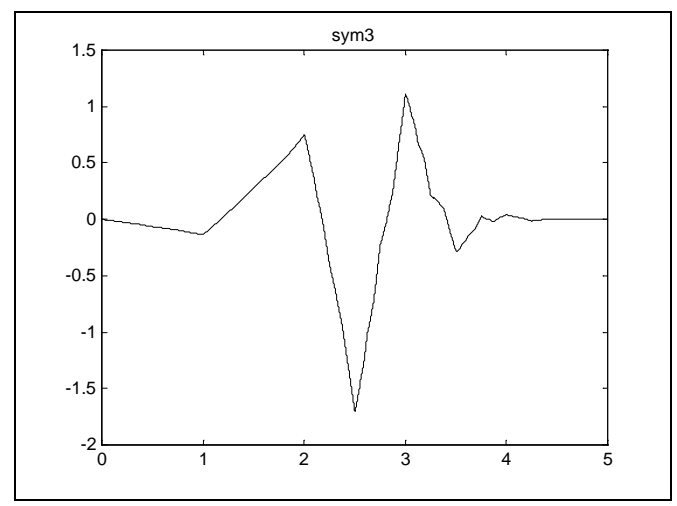

Figura 2: Função Wavelet-mãe: Symlet 3

tas, tanto para métodos baseados nos componentes de alta frequiência quanto nos métodos baseados em componentes de freqüência fundamental.

A análise por wavelet permite localizar com precisão os instantes de tempo da ocorrência de eventos, pontos de descontinuidades, etc, o que a torna muito adequada para a aplicação na localização de faltas, quando da utilização dos métodos baseados em ondas viajantes.

Este trabalho faz uso da técnica de AMR, onde o sinal a ser analisado é decomposto em níveis de resolução. Com isso, obtemos os sinais de aproximação (A1) e detalhe (D1) relativos ao sinal original. Analisando o sinal de detalhe 1, o qual representa uma faixa de alta freqüência do sinal em análise, o mesmo será capaz de revelar o instante de chegada das ondas nos terminais da linha de transmissão.

Neste trabalho optou-se pelo uso da wavelet-mãe symlet 3 (sym3), por esta melhor se adaptar as formas de onda que caracterizam o problema delineado. Como ilustração, visualiza-se a forma de onda característica da wavelet-mãe symlet 3 na Figura 2.

\section{O MÉTODO DE LOCALIZAÇÃO DE FAL- TAS PROPOSTO}

Como anteriormente mencionado, o método de localização de faltas desenvolvido é fundamentado no princípio das ondas viajantes de tensão geradas por uma situação de falta. Este princípio pode ser melhor entendido através do diagrama Lattice, ilustrado na Figura 3, onde foi considerado uma linha de transmissão de comprimento $l$, conectada entre os barramentos $A$ e $B$.

Caso ocorra uma falta a uma distância $d$ do barramento $A$, esta aparecerá como uma abrupta injeção de tensão ou corrente no ponto de falta. Esta injeção viajará como uma onda

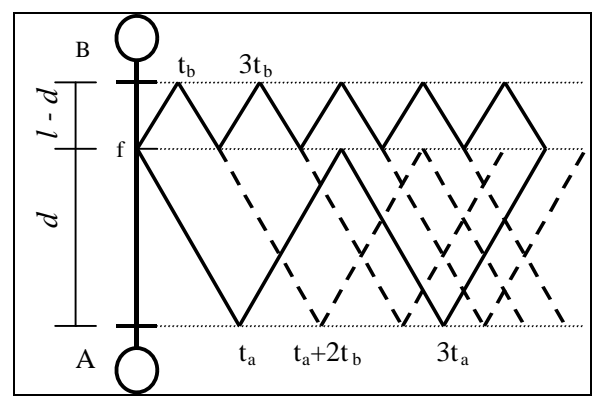

Figura 3: Diagrama Lattice

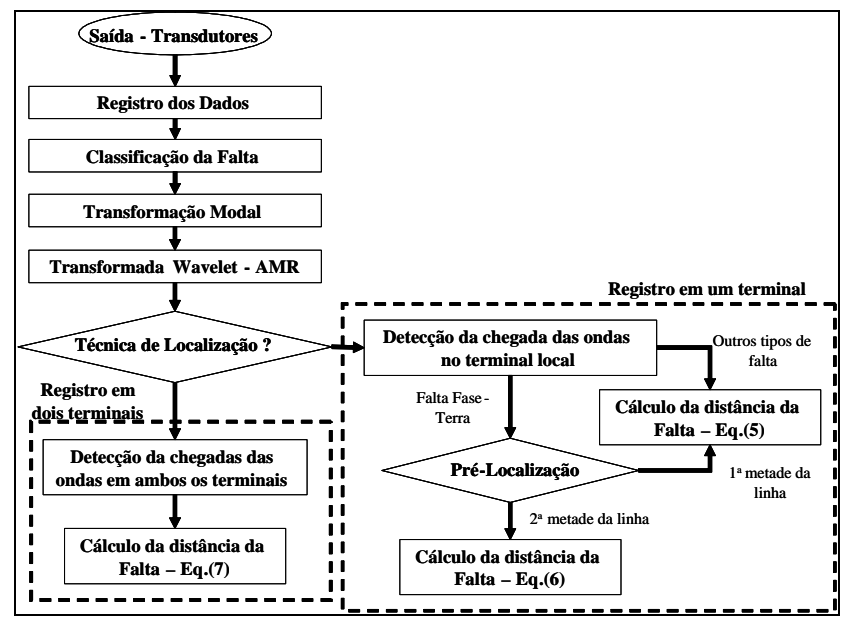

Figura 4: Fluxograma do algoritmo de localização de faltas

ao longo da linha em ambas as direções. Ao encontrar uma descontinuidade (terminais da linha) esta onda se reflete e volta ao ponto de falta onde sofrerá novas reflexões e assim sucessivamente até ser alcançado o regime permanente de falta. Desse modo, os transitórios gerados pela falta registrados nos terminais da linha conterão abruptas mudanças em intervalos comensurados com o tempo de viagem dos sinais entre o ponto de falta e os terminais da linha. Portanto, conhecendo-se o intervalo de tempo de viagem da onda do ponto de falta aos terminais da linha e a velocidade de propagação da onda na linha em questão, a distância do ponto da falta a um terminal de referência poderá ser facilmente estimada.

Baseado no exposto, foi desenvolvido um algoritmo computacional destinado à localização de faltas, dispondo-se da teoria de ondas viajantes e uso da TW, para detectar a chegada das ondas nos terminais. A Figura 4 ilustra o fluxograma do algoritmo de localização proposto.

Pode-se notar, pela análise do fluxograma apresentado na Figura 4, que o algoritmo é composto por duas técnicas de lo- 
calização de faltas que se diferenciam pela de aquisição dos dados, as quais apresentam uma parte comum para condicionamento do sinal. Os estágios que compõem o algoritmo serão descritos a seguir.

\subsection{Registro dos Dados}

Os dados a serem analisados podem ser obtidos a partir de um ou de dois terminais da linha conforme a disponibilidade dos mesmos. Os sinais de alta freqüência de corrente podem ser obtidos diretamente através dos transformadores de corrente convencionais, entretanto, devido a limitação da banda de passagem dos transformadores de potencial convencionais, os sinais de alta freqüência de tensão devem ser medidos por meio transdutores especiais de tensão, conforme (Bo et al., 1997 e 2000). Ressalta-se que para o processamento da localização da falta foram utilizados apenas os sinais da forma de onda da tensão registrados ora em um, ora em ambos os terminais da linha. Quanto aos sinais de corrente, esses são necessários apenas quando da utilização da técnica de dados de somente um terminal, para que se possa classificar a falta conforme será evidenciado no próximo item.

\subsection{Classificação da Falta}

A classificação da falta é realizada através da comparação dos fasores trifásicos de corrente e de sequiência zero. Quando se utilizam dados de dois terminais, a classificação da falta pode ser facultativa, deixando claro que esta é muito importante para que se possa prever a gravidade da falta e preparar adequadamente a equipe de manutenção e reparo. Por outro lado, utilizando-se de dados de apenas um terminal, a classificação da falta se torna essencial, visto que é necessário distinguir dentre faltas fase-terra dos outros tipos de faltas, como será posteriomente evidenciado.

\subsection{Transformação Modal}

Em sistemas trifásicos, a propagação dos sinais transitórios pode ser melhor observada desacoplando as fases em seus componentes modais, aplicando-se a técnica de transformação modal. A transformação modal permite que um sistema trifásico seja tratado como um sistema com três circuitos monofásicos independentes. Os valores de fase são transformados em três modos desacoplados: um modo terra (modo 0) e dois modos aéreos (modos 1 e 2), cada qual apresentando características próprias.

O algoritmo desenvolvido faz uso apenas dos sinais do modo aéreo 1 e do modo terra. $\mathrm{O}$ sinal do modo aéreo 1, como está presente em todos os tipos de faltas é essencialmente utilizado para o cálculo da distância, enquanto o sinal do modo terra é utilizado ocasionalmente, em casos de faltas fase- terra, para pré-localizar a falta. Nesta análise, os modelos das linhas foram considerados totalmente transpostos, possibilitando desta forma empregar a matriz de transformação de Clarke, (Clarke, 1943). É importante ressaltar que apenas os sinais de tensão são desacoplados conforme a Equação 3.

$$
\left[\begin{array}{l}
V_{0} \\
V_{1} \\
V_{2}
\end{array}\right]=\frac{1}{3} \cdot\left[\begin{array}{lll}
1 & 1 & 1 \\
2 & -1 & -1 \\
0 & \sqrt{3} & -\sqrt{3}
\end{array}\right] \cdot\left[\begin{array}{l}
V_{a} \\
V_{b} \\
V_{c}
\end{array}\right]
$$

Onde $\mathrm{V}_{0,1,2}$; são os vetores modais de tensão e $\mathrm{V}_{a, b, c}$ são os vetores de fase da tensão.

A velocidade de propagação do modo terra $\left(\mathrm{V}_{0}\right)$ e do modo aéreo $1\left(\mathrm{~V}_{1}\right)$ são dadas respectivamente por:

$$
\begin{aligned}
v_{0} & =\frac{1}{\sqrt{\left(L_{0} C_{0}\right)}} \\
v_{1} & =\frac{1}{\sqrt{\left(L_{1} C_{1}\right)}}
\end{aligned}
$$

\subsection{Análise Multiresolução}

Considerando a excelente capacidade da TW em discriminar e identificar com precisão os instantes de descontinuidade sobre formas de ondas, a ferramenta mostra-se propícia à aplicação na localização de faltas em sistemas elétricos. Tal localização é possível em virtude da TW detectar os instantes de reflexão das ondas nos terminais considerados.

Os sinais modais de tensão, modo aéreo 1 e em certas ocasiões modo terra, são decompostos em dois níveis através da análise multiresolução, utilizando como wavelet mãe a symlet3 (sym3). Esta foi escolhida como wavelet mãe por melhor representar o problema de localização, evidenciada através de testes comparando-se as wavelets da família Daubechies de ordem 2 à 10 com as da Symlet de ordem 2 à 8.

Uma vez decompostos os sinais modais, foram utilizados pelo algoritmo apenas os sinais dos coeficientes wavelets (CWs) de detalhe um, ou simplesmente detalhe 1 (D1), referente ao modo 1 e ao modo terra, sendo que os mesmos compreendem a faixa de frequiência de $60-30 \mathrm{kHz}$. Por estes coeficientes, pode-se caracterizar os instantes de reflexão das ondas viajantes do ponto de falta aos barramentos, necessários para estimação da falta. É importante lembrar que, para o cálculo da distância da falta, será essencialmente usado o tempo de reflexão das ondas, obtido através dos sinais de detalhe 1 do modo aéreo 1 . Durante os testes também se considerou a utilização do sinal de detalhe 2 (D2), entretanto, não foram alcançados resultados satisfatórios, basicamente por estes sinais conterem uma faixa de freqüência não muito adequada para o método proposto.

Além disso, os sinais dos $\mathrm{CW}$ de detalhe 1 e 2 de ambos os modos (modo 0 e modo 1 ) são elevados ao quadrado com 
o intuito de minimizar o ruído presente no sinal, como proposto em Santoso et al. (1996).

\subsection{Técnicas de Localização}

$\mathrm{O}$ algoritmo proposto foi desenvolvido de modo a permitir ao usuário definir a técnica de localização a ser empregada conforme o modo de aquisição de dados disponível e que melhor se adapte ao seu sistema. Duas técnicas são oferecidas, as quais se diferenciam pela maneira de aquisição dos dados provindos de (i) apenas um terminal ou de (ii) dois terminais. Contudo, utilizando-se da técnica de localização com dados registrados em ambos os terminais, torna-se necessário que haja um meio de comunicação entre os terminais para a transferência dos dados, bem como um método para a sincronização das medições nos mesmos.

Para atender ao problema de sincronização de dados, temos como proposta a utilização do GPS (Global Positioning Satellite) como apresentado em (Kim et al., 2001; Zhao et al, 2001), no qual um sinal de tempo de referência, transmitido via satélite, fica disponível nos diversos pontos uma rede elétrica, permitindo que os sinais sejam sincronizados mediante a um esquema de comunicação entre os terminais da linha. Em cada terminal, para cada amostra dos sinais, poderá ser armazenado o tempo real em que ela foi obtida, utilizando a referência de tempo obtida do GPS, de forma que um atraso na chegada dos dados do terminal remoto ao terminal local, poderá ser corrigido verificando-se os instantes em que as amostras foram obtidas.

Quanto ao meio de comunicação para transferência dos dados entre os dois terminais, pode-se empregar um que não exija tanta velocidade e canais de comunicação contínuos, uma vez que o nosso localizador opera off-line. A comunicação dos dados pode ser feita via rádio, satélite, modem ou microondas. Outro meio de transmissão de dados que vem sendo utilizado atualmente é a fibra óptica no interior dos cabos guarda (Urusawa et al., 1989), conhecido como OPGW (do inglês, Fiber Optic Overhead Ground Wire), que garante alta velocidade de transmissão e imunidade à interferências eletromagnéticas.

\subsection{Localização de faltas utilizando da- dos de um terminal}

Esta técnica faz uso de dados registrados apenas no terminal local, não necessitando de canais de comunicação ou meios de sincronização dos dados. As etapas do algoritmo que compõem esta técnica serão descritas no que segue.

\subsubsection{Detecção do instante de chegada das ondas no terminal local}

A detecção do instante da chegada das ondas no terminal local é feita através da análise dos sinais do detalhe 1 do modo aéreo 1 e pela comparação destes, a limiares auto-ajustáveis definidos, ou seja, caso o sinal do detalhe 1 exceda a um limiar previamente estabelecido, este caracterizará a chegada de uma onda e seu respectivo instante de tempo será registrado. Este processo se repete até a detecção da chegada da segunda onda viajante no terminal local.

\subsubsection{Pré-localização da falta}

Este estágio é peculiar a técnica que utiliza dados de um terminal, pois em casos de faltas fase-terra, observou-se que as reflexões provenientes do terminal remoto não podem ser desprezadas. Portanto, dependendo da localização da falta fase-terra caracterizada, a chegada da segunda onda viajante observada no terminal local pode ser proveniente ou do ponto de falta ou do terminal remoto, o que conduz a equacionamentos diferentes para a localização da mesma. Caso a falta fase-terra incida na primeira metade da linha coberta pelo localizador, a chegada da segunda onda será proveniente do ponto de falta. A recíproca não é verdadeira para faltas faseterra que incidam na segunda metade da linha. Neste caso a segunda reflexão a ser registrada no terminal local é proveniente do terminal remoto. Portanto, em vista do exposto, para casos de faltas fase-terra, é necessário que seja efetuada uma pré-localização da falta, ou seja, determinar se a falta ocorreu na primeira ou segunda metade da linha, para que se proceda ao cálculo correto da distância da mesma. A pré-localização da falta é baseada na comparação da diferença entre os tempos das reflexões iniciais revelados pela TW com relação ao modo terra e modo aéreo 1 , com a diferença entre os tempos de propagação do modo terra e aéreo 1 com relação ao meio da linha coberta pelo localizador, como proposto por Abur e Magnago (2000). Em outras palavras, quanto maior a distância da falta, maior será a diferença entre os tempos de propagação das primeiras reflexões reveladas pela TW com relação a ambos os modos. Caso esse valor seja maior que a diferença dos tempos de reflexão para a metade da linha, a falta estará alocada na segunda metade, caso contrário, a falta estará alocada na primeira metade da mesma.

Para os demais tipos de falta, procede-se diretamente ao cálculo da distância da falta sem a necessidade da prélocalização da mesma.

\subsubsection{Cálculo da distância da falta}

Com base na Figura 3 e com referência ao terminal $A$, para faltas fase-terra na primeira e segunda metade da linha, a distância da falta pode ser estimada conforme as Eqs.(5) e (6) 
respectivamente. Para os demais tipos de falta, a distância da falta é estimada somente pela aplicação da Eq.(5).

$$
\begin{gathered}
d=\frac{v_{1} \cdot\left(3 t_{a}-t_{a}\right)}{2} \\
d=l-\frac{v_{1} \cdot\left(\left(t_{a}+2 t_{b}\right)-t_{a}\right)}{2}
\end{gathered}
$$

Onde: $d$ é a distância da falta $(\mathrm{km}) ; v_{1}$ a velocidade de propagação da onda na linha em análise referente ao modo aéreo $1 \mathrm{em}(\mathrm{km} / \mathrm{s}) ; l$ o comprimento total da linha coberta pelo localizador e $t_{a}$ e $t_{b}$ os tempos de viagem da onda do ponto de falta ao terminal $A$ e $B$ respectivamente.

\subsection{Localização de faltas utilizando da- dos de dois terminais}

Esta técnica faz uso de dados registrados nos dois terminais para processar a localização da falta. Porém, como já mencionado, a mesma necessita de um canal de comunicação entre os terminais para a transmissão dos dados, assim como de um meio de sincronização dos dados, o que a torna mais onerosa, caso o sistema em questão não tenha disponível estes requisitos. Por outro lado, esta técnica apresenta um algoritmo bem mais simplificado e preciso, como poderá ser constatado no decorrer desta seção.

No que segue, serão apresentados os estágios que constituem a técnica que utiliza dados registrados em dois terminais, conforme o fluxograma apresentado na Figura 4.

\subsubsection{Detecção do instante de chegada das ondas em ambos os terminais}

Nesta técnica, a detecção do instante de chegada das ondas em ambos os terminais é feita de modo análogo ao item 3.6.1, ou seja, através da comparação do sinal do detalhe 1 , do modo aéreo 1, registrado nos dois terminais, à limiares autoajustáveis. Porém, nesta técnica, detecta-se apenas o instante de chegada da primeira onda em cada um dos dois terminais.

\subsubsection{Cálculo da distância da falta}

Com base na Figura 3 e referência ao terminal $A$, a distância da falta pode ser estimada conforme (Eq.(7))

$$
d=\frac{l-v \cdot\left(t_{b}-t_{a}\right)}{2}
$$

sendo as variáveis envolvidadas já apresentadas no item 3.6.3.

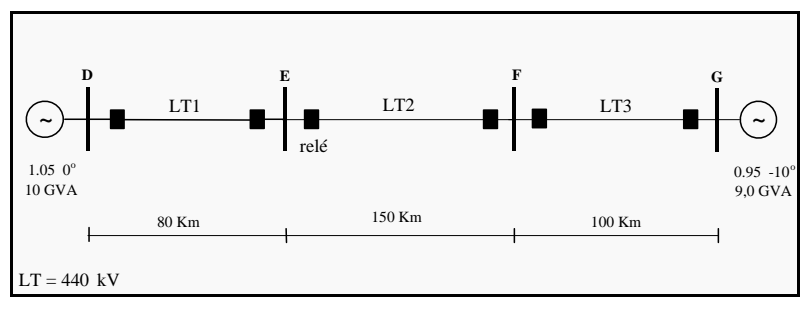

Figura 5: Sistema de transmissão simples horizontal

\section{SISTEMAS ANALISADOS}

Para validação da abordagem proposta, foi utilizado um modelo de linha de transmissão de $440 \mathrm{kV}$, com $330 \mathrm{~km}$ de comprimento, considerando-se a linha com parâmetros distribuídos e totalmente transposta. A topologia do sistema elétrico analisado em todo o processo é a representada na Figura 5. A estrutura da linha de transmissão corresponde a uma linha típica da CESP (Companhia Energética de São Paulo), empregada entre as cidades de Araraquara - Bauru e Jupiá - Ilha Solteira.

Os dados dos sinais faltosos foram obtidos utilizando-se o conceituado software ATP - Altenative Transients Program, levando-se em conta vários tipos de falta (fase-terra, fasefase, fase-fase-terra e trifásicas) em diferentes localizações ao longo da linha $(10,25,40,55,70,75,80,95,110,125$ e $140 \mathrm{~km}$ entre os barramentos $E$ e $F$ ), com diferentes ângulos de incidência $\left(0\right.$ e $\left.90^{\circ}\right)$ e resistências de falta $(0,50$ e $100 \Omega)$.

Além do circuito simples apresentado, uma topologia de circuito duplo vertical também foi explorada (Figura 6), sendo os resultados apresentados posteriormente.

Cabe ressaltar que todas as situações de faltas foram geradas a uma taxa amostral de $120 \mathrm{kHz}$. Os valores analisados correspondem aos valores trifásicos dos sinais de tensões e correntes do sistema, tomados com respeito ao barramento $E$ e quando necessário, do barramento $F$. A área de cobertura do localizador corresponde apenas a linha entre os barramentos $E$ e $F$, ou seja, a linha de $150 \mathrm{~km}$.

Durante os testes também buscou-se avaliar a influência de

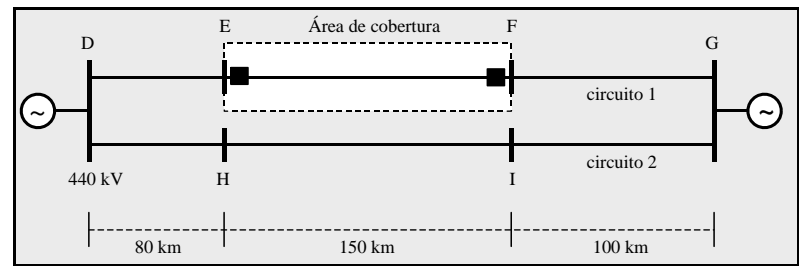

Figura 6: Sistema de transmissão duplo vertical 
diversos outros fatores como: taxa amostral utilizada, alta resistência de falta ( 200 e $400 \Omega$ ), falta de sincronização dos dados (quando utilizado dados de dois terminais), imprecisão dos parâmetros da linha e acoplamento mútuo entre fases de um sistema duplo vertical.

Vale destacar que da combinação entre as variáveis apresentadas, um conjunto com mais de 2000 diferentes situações faltosas foram aplicadas com o objetivo de testar e validar o método proposto.

\section{AVALIAÇÃO DO ALGORITMO DE LO- CALIZAÇÃO PROPOSTO}

Nesta seção são apresentados os resultados gerais alcançados pelo algoritmo de localização de faltas proposto, implementado em ambiente Matlab. Os resultados estão dispostos conforme a técnica de localização utilizada e o sistema de transmissão caracterizado.

\subsection{Resultados obtidos utilizando dados obtidos em um terminal}

A Figura 7 ilustra os sinais do detalhe 1 referentes ao modo aéreo 1 , considerando uma falta fase-terra aplicada a $110 \mathrm{~km}$ do barramento $E$, com ângulo de incidência de $0^{\circ}$ e resistência de falta de $50 \mathrm{ohms}$. Pode-se observar a habilidade da TW em detectar a chegada das ondas no terminal local. Neste caso a distância estimada pelo algoritmo foi de 109,7 $\mathrm{km}$.

As Figuras 8 e 9 ilustram os resultados de algumas situações de faltas fase-fase-terra onde se variou a distância e a resistência de falta. Pela Figura 8 pode-se observar que para variações da resistência de falta em 0,50 e $100 \Omega$, a maioria dos erros constatados ficaram em torno de $1,5 \%$ do comprimento da linha, e que a variação da distância e da resistência de falta (linhas exatamente sobrepostas), não influenciaram a precisão da técnica de localização utilizada, mesmo para altas resistências de falta. A Figura 9, refere-se ao mesmo teste avaliado na Figura 8, porém nesta nova situação foram

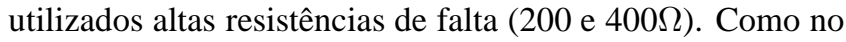
caso anterior, pode-se constatar o mesmo desempenho do algoritmo frente a situações faltosas consideradas críticas.

A Figura 10 ilustra situações de faltas fase-terra testadas para variações do ângulo de incidência da falta de 0 e $90^{\circ}$. Pela análise dos resultados apresentados, observa-se que maioria dos erros ficaram em torno de 1,0\%. Deste fato, pode-se concluir que o efeito da variação do ângulo de incidência da falta é praticamente insignificante quando da ultilização de dados registrados em um único terminal.

A Figura 11 ilustra um outro tipo de teste onde buscou-se

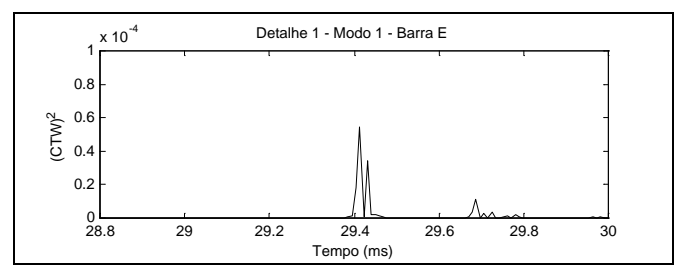

Figura 7: Detalhe 1: Falta fase-terra registrada na barra E

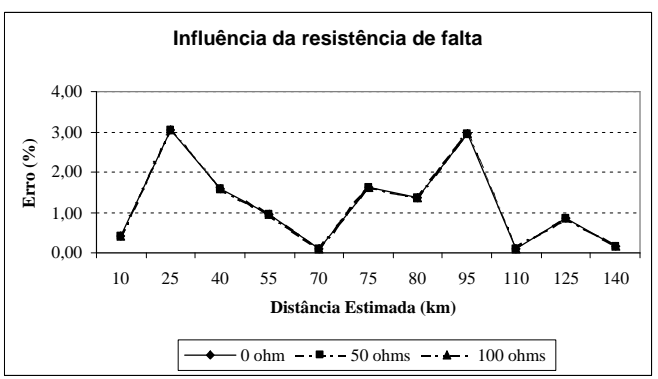

Figura 8: Faltas fase-fase-terra com ângulo de incidência de $0^{\circ}$

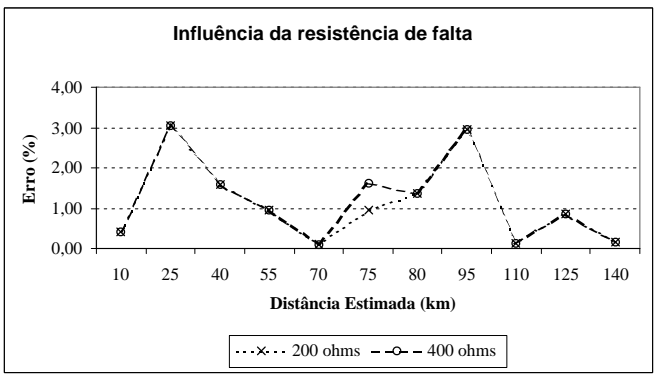

Figura 9: Faltas fase-fase-terra com ângulo de incidência de $0^{\circ}$ e elevada resistência de falta

avaliar o comportamento do algoritmo frente aos diversos tipos de faltas. Observa-se por estes resultados que no caso de faltas fase-terra e trifásicas o algoritmo mostra-se ligeiramente mais preciso que para os outros dois tipos de falta (fase-fase-terra e fase-fase), que por sua vez apresentaram resultados semelhantes, o que se confirma pela sobreposição dos resultados apresentados na Figura 11. Do exposto, independente do tipo de falta considerado, pode-se afirmar que o algoritmo proposto não apresenta problemas relacionados a variável apontada.

Durante os testes também buscou-se verificar a influência na precisão do algoritmo, utilizando dados de um terminal, quando da entrada de parâmetros imprecisos da linha de transmissão em questão (valores relacionados a indutância e a capacitância), uma vez que estes parâmetros influem diretamente na determinação da velocidade de propagação da 


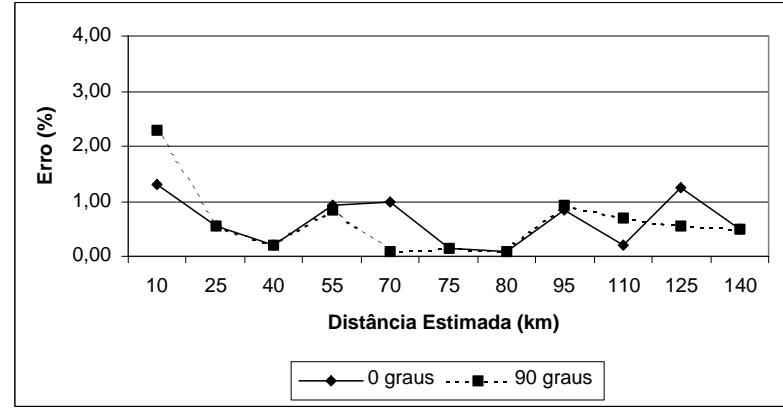

Figura 10: Falta fase-terra com ângulo de incidência de $0^{\circ}$

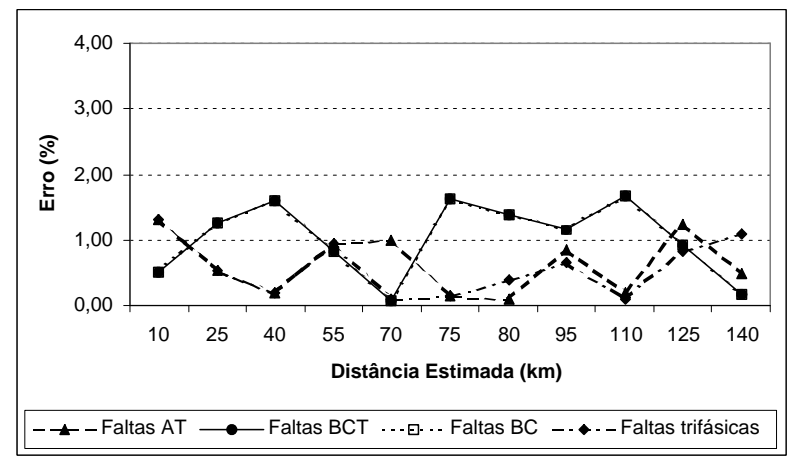

Figura 11: Faltas fase-terra, fase-fase-terra, fase-fase e trifásicas, com ângulo de incidência de $0^{\circ}$ e resistência de falta de $0 \Omega$

onda na linha e consequentemente na estimação da localização da falta. A Figura 12 ilustra alguns resultados que caracterizam o fornecimento de parâmetros imprecisos da linha de transmissão ao algoritmo implementado (índice de imprecisão considerado: $\pm 5 \%$ ). Pela análise destes, confirma-se a influência da variação de tais parâmetros na precisão do algoritmo quando utilizado dados de um terminal. Contudo, apesar desta imprecisão, os resultados ainda mostram-se satifatórios à sua aplicação, registrando erros inferiores a 3,0\%.

Além de verificar a aplicabilidade do algoritmo proposto sobre uma linha de transmissão em circuito simples horizontal, utilizou-se de outro sistema de transmissão, em circuito duplo vertical, como exposto anteriormente. A Figura 13 ilustra a comparação de testes feitos nos circuitos simples e duplo. Pela análise desta, verifica-se que o algoritmo é aplicável a diferentes sistemas de transmissão e que o mesmo não sofre influência significativa do acoplamento mútuo entre fases, como caracterizado pelo circuito de transmissão duplo vertical comentado.

As Tabelas 1 e 2 ilustram os resultados gerais referentes a todas as situações de testes avaliadas, com o intuito de validar a técnica de localização de faltas que se utiliza de dados registrados em apenas um terminal. Estas tabelas diferenciam-se pelo tipo de linha de transmissão utilizada: circuito simples ou circuito duplo, respectivamente. Através destas, podese constatar o bom desempenho da abordagem proposta. Ressalta-se que nestes testes, considerou-se apenas a variação dos seguintes parâmentros: distância da falta, ângulo de incidência e a resistência de falta.

Atenta-se ao fato de que os resultados obtidos frente aos testes realizados sobre o circuito duplo podem sofrer pequenas modificações em virtude do número de testes realizados entre os dois sistemas, uma vez que o sistema simples horizontal foi alvo da maioria dos testes direcionados a esta aplicação.

Cabe ressaltar que a técnica que utiliza dados de um terminal é menos onerosa, porém, mais complexa devido a necessidade de se detectar a chegada da primeira e segunda onda no terminal local e da necessidade de se classificar o tipo de falta em análise para se proceder a pré-localização da mesma.

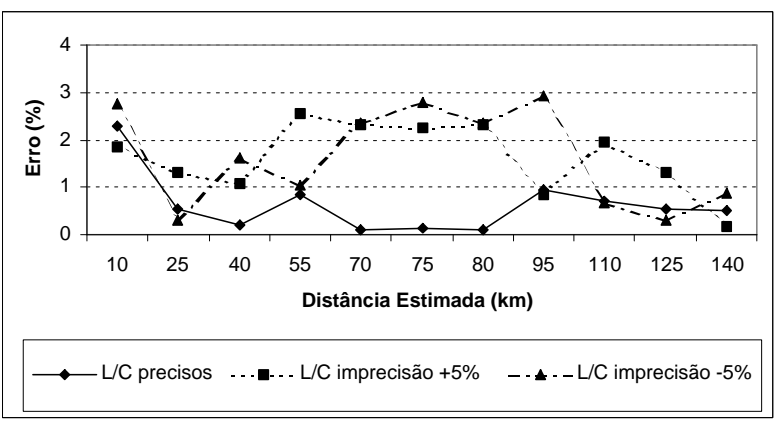

Figura 12: Influência da imprecisão dos parâmetros da linha

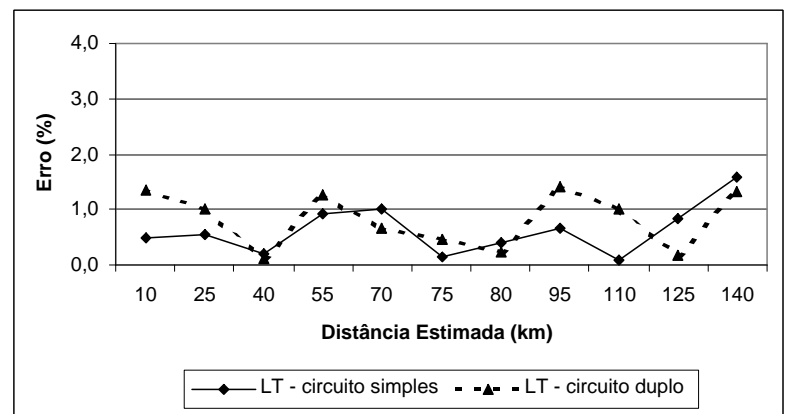

Figura 13: Influência do acoplamento mútuo entre fases de um circuito duplo - faltas fase-fase 
Tabela 1: Resultados médios gerais obtidos sob o circuito simples

\begin{tabular}{|l|c|c|}
\hline \multicolumn{1}{|c|}{$\begin{array}{c}\text { Tipo da } \\
\text { Falta }\end{array}$} & No. de Situações & $\begin{array}{c}\text { Erro Médio } \\
(\mathbf{\%})\end{array}$ \\
\hline Fase-terra & 308 & 1,51 \\
\hline Fase-fase-terra & 242 & 0,99 \\
\hline Fase-fase & 110 & 0,94 \\
\hline Trifásicas & 22 & 0,71 \\
\hline
\end{tabular}

Tabela 2: Resultados médios gerais obtidos sob o circuito duplo

\begin{tabular}{|l|c|c|}
\hline \multicolumn{1}{|c|}{$\begin{array}{c}\text { Tipo da } \\
\text { Falta }\end{array}$} & No. de Situações & $\begin{array}{c}\text { Erro Médio } \\
(\boldsymbol{\%})\end{array}$ \\
\hline Fase-terra & 66 & 1,12 \\
\hline Fase-fase-terra & 66 & 1,11 \\
\hline Fase-fase & 22 & 1,11 \\
\hline Trifásicas & 22 & 0,98 \\
\hline
\end{tabular}

\subsection{Resultados obtidos utilizando dados de dois terminais}

A Figura 14 ilustra a chegada das primeiras ondas de tensão em ambos os terminais da linha reveladas pelos sinais do detalhe 1 , referentes ao modo aéreo 1, para uma situação de falta fase-terra, aplicada a $110 \mathrm{~km}$ do barramento $E$, com ângulo de incidência de $0^{\circ}$ e resistência de falta de 100 ohms. Neste caso a distância estimada pelo algoritmo foi de 109,92 $\mathrm{km}$.

$\mathrm{O}$ algoritmo proposto utilizando dados registrados em ambos os terminais da linha foi avaliado de modo análogo a técnica
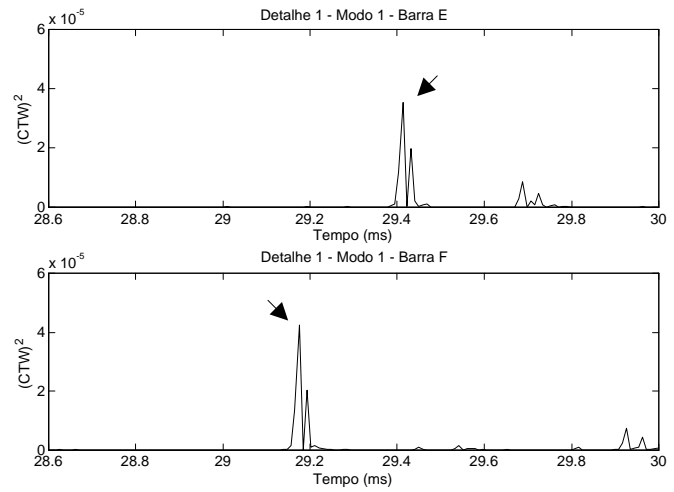

Figura 14: Detalhe 1: Falta fase-terra registrada nos barramentos E e F

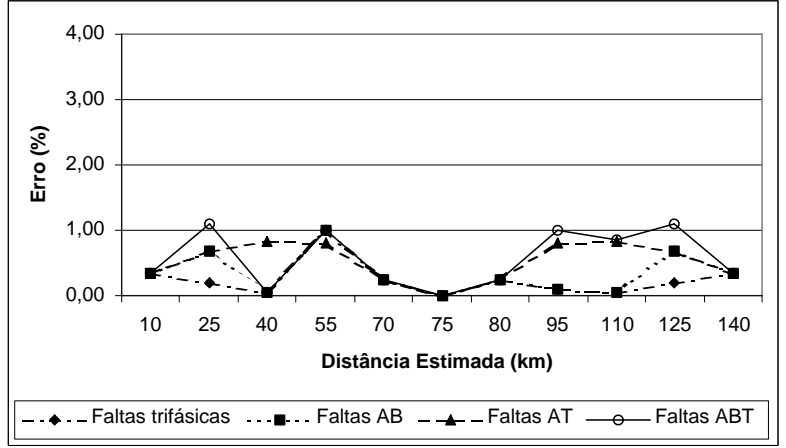

Figura 15: Faltas fase-terra,fase-fase-terra, fase-fase e trifásicas com ângulo de incidência de $90^{\circ}$ e resistência de falta de $0 \Omega$

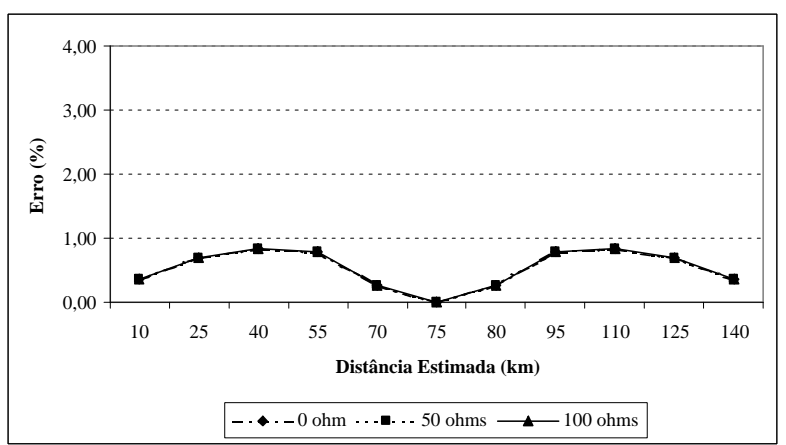

Figura 16: Faltas fase-terra com ângulo de incidência de $90^{\circ}$

que utiliza dados de um terminal, diferenciando-se apenas entre algumas situações cabíveis somente a técnica que utiliza dados de dois terminais. No que segue serão ilustrados alguns testes elaborados para avaliação do método proposto.

A Figura 15 ilustra situações onde variou-se o tipo de falta aplicada. Pode-se verificar que a maioria dos erros variam até $1,0 \%$. Constata-se também que o algoritmo que dispõe de dados provenientes de dois terminais, não é significantemente influenciado pelo tipo de falta aplicado, salvo condições semelhantes entre as mesmas.

Quanto aos testes realizados para verificar a influência da resistência de falta, Figuras 16 e 17, novamente pode-se afirmar que a precisão da técnica em análise não é significantemente influênciada pela resitência de falta, mesmo quando da presença de elevadas resistências de terra. A grande maioria dos erros ficaram restritos ao valor máximo de $1,0 \%$.

O gráfico ilustrado pela Figura 18 demonstra o comportamento da técnica em análise frente a variações da taxa de amostragem do sinal. Como esperado, confirma-se que a precisão do método é dependente da taxa amostral e que para 


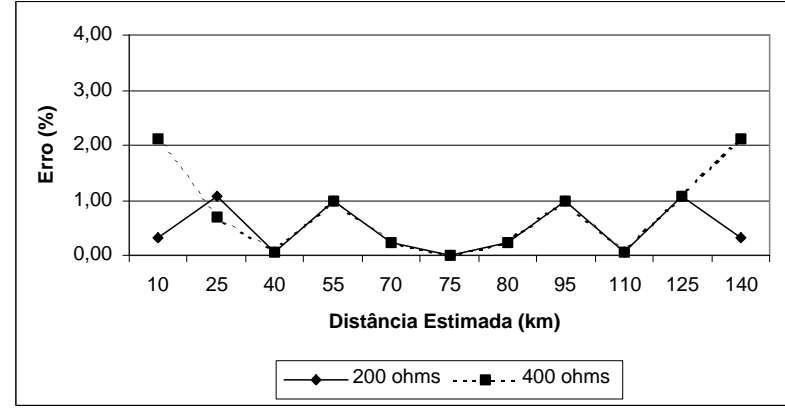

Figura 17: Faltas fase-terra com ângulo de incidência de $90^{\circ}$ e elevadas resistências de falta

taxas inferiores $120 \mathrm{kHz}$, o mesmo apresenta um decréscimo na sua precisão. Essas mesmas observações quanto a variação da taxa amostral são validas para a técnica que trabalha com dados de um terminal.

Quando se trabalha com dados de dois terminais, é necessário que os dados registrados em ambos os terminais sejam perfeitamente sincronizados, para que se proceda a um perfeita estimação da distância da falta. Logo, buscou-se durante os testes verificar a influência do dissincronismo dos dados sobre a precisão da técnica em análise. A Figura 19, ilustra um teste onde foi considerado um erro de sincronismo equivalente a uma amostra na frequência de $120 \mathrm{kHz}$, o que corresponde a um tempo de $8,33 \mu \mathrm{s}$. Observa-se pela mesma Figura 19 que realmente um dissincronismo dos dados pode decrementar a precisão do método. Contudo, considerandose que esta imprecisão seja mínima, a resposta do algoritmo ainda permanece satisfatória.

A Figura 20 ilustra alguns testes onde buscou-se verificar a influência da imprecisão dos parâmetros da linha sobre a técnica em análise. Como esperado, confirma-se a influência da variação de tais parâmetros na precisão do algoritmo quando da utilização de dados registrados em dois terminais, porém os resultados ainda mostram-se satifatórios e melhores que os obtidos pela técnica que utiliza dados de um terminal.

Durante os testes, também se avaliou o desempenho da técnica que utiliza dados de dois terminais ao ser aplicada sobre um sistema de transmissão duplo vertical. Alguns destes resultados pode ser visualizado através da Figura 21. Podese afirmar com base na mesma figura que a técnica em análise pode ser utilizada em outras configurações de linhas de transmissão sem prejuízo da sua precisão. Além disso, podese também afirmar que a técnica utilizada é quase imune ao efeito do acoplamento mútuo entre fases de um circuito duplo de transmissão.

As Tabelas 3 e 4 ilustram os resultados gerais referentes a to-

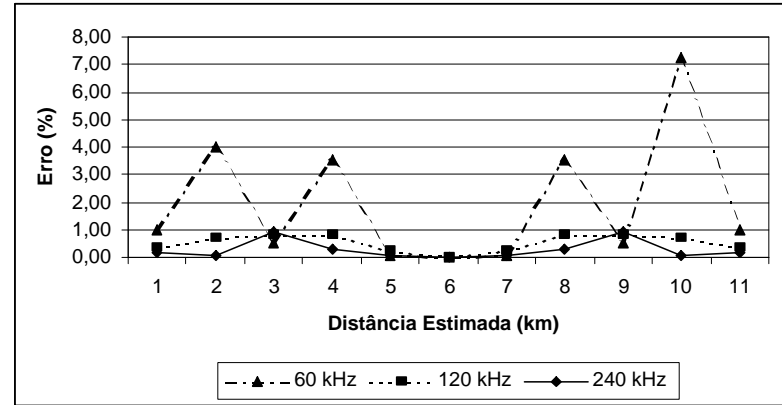

Figura 18: Influência da taxa amostral do sinal utilizado

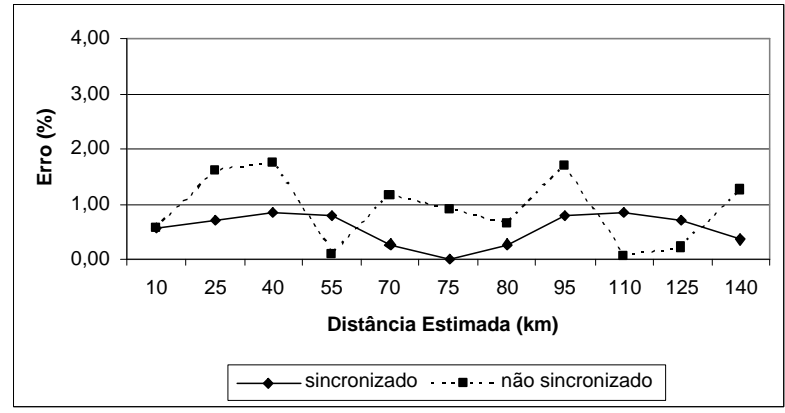

Figura 19: Influência da falta de sincronismo dos dados $8,33 \mu$ s (uma amostra a $120 \mathrm{kHz}$ )

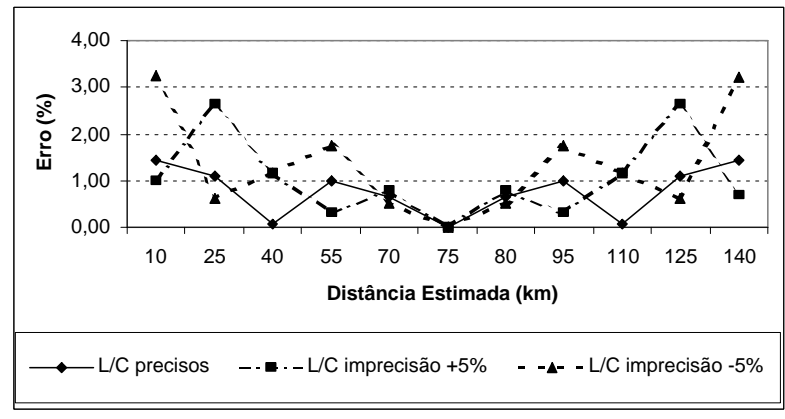

Figura 20: Influência da imprecisão dos parâmetros da linha

dos os testes aplicados, onde variou-se apenas o tipo da falta, a distância, o ângulo de incidência e a resistência de falta, com o intuito de avaliar a técnica de localização de faltas que utiliza dados registrados em dois terminais. Através das mesmas, pode-se avaliar o bom desempenho do método proposto. Novamente, estas tabelas diferenciam-se entre o tipo de linha de transmissão utilizada nas simulações, seja por um circuito simples ou por um circuito duplo, respectivamente.

Um maior detalhamento sobre a metodologia aqui proposta, bem como dos resultados alcançados pela mesma, pode ser 


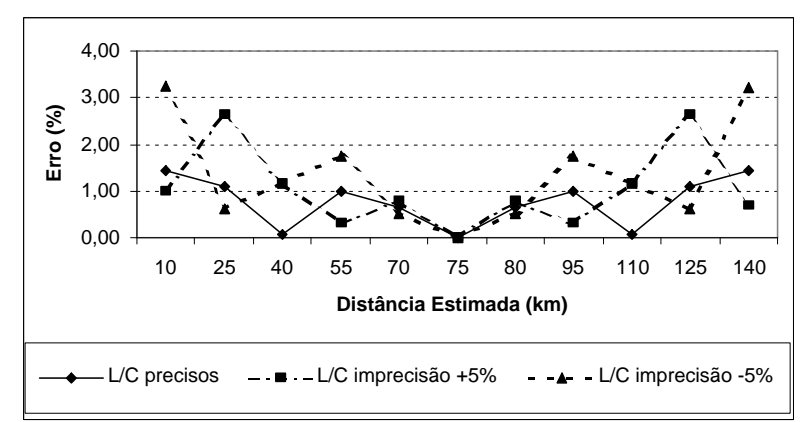

Figura 21: Influência do acoplamento mútuo entre fases de um circuito duplo

Tabela 3: Resultados médios gerais obtidos sob o circuito simples

\begin{tabular}{|l|c|c|}
\hline \multicolumn{1}{|c|}{$\begin{array}{c}\text { Tipo da } \\
\text { Falta }\end{array}$} & No. de Situações & $\begin{array}{c}\text { Erro Médio } \\
(\boldsymbol{\%})\end{array}$ \\
\hline Fase-terra & 308 & 0,82 \\
\hline Fase-fase-terra & 242 & 0,81 \\
\hline Fase-fase & 66 & 0,70 \\
\hline Trifásicas & 22 & 0,34 \\
\hline
\end{tabular}

Tabela 4: Resultados médios gerais obtidos sob o circuito duplo

\begin{tabular}{|l|c|c|}
\hline \multicolumn{1}{|c|}{$\begin{array}{c}\text { Tipo da } \\
\text { Falta }\end{array}$} & No. de Situações & $\begin{array}{c}\text { Erro Médio } \\
(\boldsymbol{\%})\end{array}$ \\
\hline Fase-terra & 66 & 0,65 \\
\hline Fase-fase-terra & 66 & 0,59 \\
\hline Fase-fase & 22 & 0,60 \\
\hline Trifásicas & 22 & 0,71 \\
\hline
\end{tabular}

obtida em Silva, 2003.

\section{CONCLUSÕES}

Este trabalho apresenta a aplicação da TW para análise dos transitórios de alta frequiência gerados por uma falta em uma linha de transmissão, com o propósito de se determinar a localização da mesma.

Como mencionado, a abordagem foi baseada na teoria das ondas viajantes, onde, uma simples análise dos instantes de chegada das ondas aos terminais da linha, reveladas pela TW, pode ser usada para estimar a localização da falta. No algoritmo geral desenvolvido, ambas as técnicas de localização empregando dados de um ou de dois terminais da linha estão disponíveis ao usuário.
Os resultados obtidos mostram que o desempenho geral do algoritmo de localização de faltas proposto foi altamente satisfatório com respeito à precisão e velocidade de resposta para todos os testes avaliados. Entretanto, deve-se ressaltar que a precisão do algoritmo utilizando dados registrados em ambos os terminais foi um pouco superior se comparados aos resultados da técnica utilizando dados de somente um terminal. O método proposto também se mostrou praticamente independente da impedância de falta, tipo da falta, ângulo de incidência da falta, posição da falta e efeito do acoplamento mútuo. Deve ser enfatizado que a taxa amostral utilizada para esta aplicação é bastante elevada quando comparada as dos localizadores e registradores digitais tradicionais, que variam de $960 \mathrm{~Hz}$ à $15360 \mathrm{~Hz}$. Entretanto, o ganho em precisão relativo a esta nova metodologia deve ser considerado.

Finalizando, pode-se afirmar que a aplicação da Transformada Wavelet ao problema de localização de faltas usando ondas viajantes mostrou-se muito adequada e promissora.

\section{AGRADECIMENTOS}

Os autores gostariam de agradecer ao Departamento de Engenharia Elétrica da Escola de Engenharia de São Carlos/USP (Brasil) pelas facilidades proporcionadas quando do desenvolvimento deste trabalho, bem como ao apoio financeiro recebido por parte da CAPES - Coordenação de Aperfeiçoamento de Pessoal de Nível Superior e da FAPESP Fundação de Amparo à Pesquisa do Estado de São Paulo.

\section{REFERÊNCIAS}

Abur, A. and Magnago, F. H. (2000). Use of Time Delays between Modal Components in Wavelet based Fault Location. ELSEVIER - Electrical Power and Energy Systems, Vol.22, pp 397-403.

Aggarwal, R. K., Coury, D. V., Johns, A. T. and Kalam, A. (1993). A Practical Approach to Accurate Fault Location on Extra High Voltage Teed Feeders. IEEE Transactions on Power Delivery, Vol.8, No.3, Jul, pp 874-883.

Bewley, L. V. (1963). Traveling Waves on Transmission Systems. John Wiley \& Sons, New York, USA.

Bo, Z. Q., Jiang, Z. C., Chen, Z. X., Dong, Z., Weller, G. and Redfern, M. A. (2000). Transients based Protection for Power Transmission Systems. IEEE - Power Engineering Society Winter Meeting, Vol. 3, Jan, pp. 1832-1837.

Bo, Z. Q., Johns, A. T. and Aggarwal, R. K (1997). A Novel Fault Locator based on the Detection of Fault Generated High Frequency Transients. IEE - Development in Power System Protection, Conference Publication, No. 434, pp 197-200. 
Clarke, E. (1943). Circuit Analysis of A-C Power Systems. John Wiley \& Sons, New York, USA.

Coury, D. V. and Gautier, L. R. (2000). Localizadores Digitais de Faltas para Linhas de Transmissão - Um Estudo Comparativo. Revista Controle e Automação, Vol. 48, p. 97-106.

Daubechies, I. (1996). Where Do Wavelets Come From? - A Personal Point of View. Proceedings of the IEEE, Vol. 84, No.4, pp 510-513, Apr.

Gale, P. F., Taylor, P. V., Naidoo, P., Hitchin, C. and Clowes, D. (2001). Traveling Wave Fault Locator Experience on Eskom's Transmission Network. IEE - Development in Power System Protection, Conference Publication, No. 479, pp 327-330.

Greenwood, A. (1971). Electrical Transients in Power Systems. Wiley-Interscience, New York.

Hwan, K. C. and Aggarwal, R. (2000). Wavelet Transform in Power Systems: Part 1 General Introduction to the Wavelet Transform. IEE - Power Engineering Journal, Vol. 14, No. 2, April, pp 81-87.

Kim, G., Kim, H. and Choi, J. (2001). Wavelet Transform based Power Transmission Line Fault Location Using GPS for Accurate Time Synchronization. IEEE/IECON: The 27th Annual Conference of the IEEE Industrial Electronics Society, pp 495-499.

Magnago, F. H. and Abur, A. Fault Location Using Wavelets. (1998). IEEE - Transactions on Power Delivery, Vol. 13, No. 4, October, pp 1475-1479.

Purushothama, G. K., Narendranath, A. U., Thukaram, D. and Parthasarathy, K. (2001). Artificial Neural Networks Applications in Fault Locators. ELSEVIER - Electrical Power and Energy Systems, Vol.23, pp 491-506.

Santoso, S.; Powers, E. J., Grady, W. M. and Hofmann, P. (1996). Power Quality Assessment via Wavelet Transform Analysis. IEEE Transactions on Power Delivery, EUA, Vol.11, No.2, pp 924-930.

Silva, M. (2003). Localização de Faltas em Linhas de Transmissão Utilizando a Teoria de Ondas Viajantes e Transformada Wavelet. Dissertação de Mestrado, Escola de Engenharia de São Carlos, Universidade de São Paulo, São Carlos - SP.

Silva, M.; Oleskovicz, M. and Coury, D. V. (2004). A fault locator for transmission lines using traveling waves and wavelet transform theory. IEE - 8th International Conference and Exhibition on Developments in Power System Protection, 5-8 April, RAI, Amsterdam, The Netherlands.
Urusawa, K., Kanemaru, K., Toyota, S. and Sugiyama, K. (1989). New fault location system for power transmission lines using composite fiber-optic overhead ground wire (OPGW). IEEE Transactions on Power Delivery, Vol.4, No.4, pp 2005-2011, Oct.

Zhang, D. J., Wu, Q. H., Bo, Z. Q. and Caunce, B. (2003). Transients Positional Protection of Transmission Lines Using Complex Wavelets Analysis. IEEE - Transaction on Power Delivery, Vol. 18, No. 3, July, pp 705-710.

Zhao, W.; Song, Y. H. and Chen, W. R. (2001). Improved GPS Traveling Wave Fault Locator For Power Cables by Using Wavelet Analysis. ELSEVIER Electrical Power and Energy Systems, Vol.23, pp 403-411. 